\title{
Discovery of Water Maser Emission in Eight AGNs with 70 m Antennas of NASA's Deep Space Network
}

\section{Citation}

Kondratko, P. T., L. J. Greenhill, J. M. Moran, J. E. J. Lovell, T. B. H. Kuiper, D. L. Jauncey, L. B. Cameron, et al. 2006. "Discovery of Water Maser Emission in Eight AGNs with 70 m Antennas of NASA's Deep Space Network." The Astrophysical Journal 638 (1) (February 10): 100-105. doi:10.1086/498641.

\section{Published Version}

10.1086/498641

\section{Permanent link}

http://nrs.harvard.edu/urn-3:HUL.InstRepos:32095369

\section{Terms of Use}

This article was downloaded from Harvard University's DASH repository, and is made available under the terms and conditions applicable to Other Posted Material, as set forth at http:// nrs.harvard.edu/urn-3:HUL.InstRepos:dash.current.terms-of-use\#LAA

\section{Share Your Story}

The Harvard community has made this article openly available.

Please share how this access benefits you. Submit a story.

\section{Accessibility}




\title{
DISCOVERY OF WATER MASER EMISSION IN EIGHT AGN WITH 70-M ANTENNAS OF NASA'S DEEP SPACE NETWORK
}

\author{
P. T. Kondratko, ${ }^{1}$ L. J. Greenhill, ${ }^{1}$ J. M. Moran, ${ }^{1}$ J. E. J. Lovell,${ }^{2}$ T. B. H. Kuiper,${ }^{3}$ D. L. Jauncey ${ }^{2}$ And L. B. \\ Cameron, ${ }^{4}$ J. F. Gómez, ${ }^{5}$ C. García-Miró, ${ }^{6}$ E. Moll, ${ }^{6}$ I. De Gregorio-Monsalvo, ${ }^{7}$ And E. Jiménez-Bailón ${ }^{7}$
}

\begin{abstract}
We report the discovery of water maser emission in eight active galactic nuclei (AGN) with the 70-m NASA Deep Space Network (DSN) antennas at Tidbinbilla, Australia and Robledo, Spain. The positions of the newly discovered masers, measured with the VLA, are consistent with the optical positions of the host nuclei to within $1 \sigma\left(0 . \prime 3\right.$ radio and $1{ }^{\prime \prime} 3$ optical $)$ and most likely mark the locations of the embedded central engines. The spectra of two sources, NGC 3393 and NGC 5495, display the characteristic spectral signature of emission from an edge-on accretion disk, with orbital velocities of $\sim 600$ and $\sim 400 \mathrm{kms}^{-1}$, respectively. In a survey with DSN facilities of 630 AGN selected from the NASA Extragalactic Database, we have discovered a total of 15 water maser sources. The resulting incidence rate of maser emission among nearby $\left(v_{\text {sys }}<7000 \mathrm{~km} \mathrm{~s}^{-1}\right)$ Seyfert $1.8-2.0$ and LINER systems is $\sim 10 \%$ for a typical rms noise level of $\sim 14 \mathrm{mJy}$ over $1.3 \mathrm{~km} \mathrm{~s}^{-1}$ spectral channels. As a result of this work, the number of nearby AGN $\left(v_{s y s}<7000 \mathrm{~km} \mathrm{~s}^{-1}\right)$ observed with $<20 \mathrm{mJy} \mathrm{rms}$ noise has increased from 130 to 449 .

Subject headings: galaxies: active — galaxies: individual (AM2158-380NED02, IC0184, NGC0235A, NGC0613, NGC3393, NGC4293, NGC5495, VIIZW073) — galaxies: Seyfert ISM: molecules — ISM: jets and outflows — masers
\end{abstract}

\section{INTRODUCTION}

Water maser emission $(\lambda=1.3 \mathrm{~cm})$ is currently the only resolvable tracer of warm dense molecular gas in the inner parsec of active galactic nuclei (AGN) and has been detected to-date in approximately 60 nuclei. In eight water maser systems that have been studied with sub-milliarcsecond resolution using Very Long Baseline Interferometry (VLBI), the mapped emission appears to trace structure and dynamics of molecular disks 0.1 to $1 \mathrm{pc}$ from supermassive black holes: Circinus (Greenhill et al. 2003), NGC 1068 (Greenhill \& Gwinn 1997), NGC 4258 (Mivoshi et al. 1995), NGC 3079 (Trotter et al. 1998), IC 2560 (Ishihara et al. 2001), NGC 5793 (Hagiwara et al. 2001), NGC 4945 (Greenhill et al. 1997), NGC 1386 (Braatz et al. 1997). Due to spatial coherence in lineof-sight velocity within the rotating structures, maser emission is detected preferentially in edge-on disks along the midline (i.e., the diameter perpendicular to the line of sight) and close to the line of sight towards the center. A characteristic spectral signature of emission from an edge-on disk thus consists of a spectral-line complex in the vicinity of the systemic velocity (low-velocity emission) and two spectral-line complexes symmetrically offset from the systemic velocity by the orbital velocity

${ }^{1}$ Harvard-Smithsonian Center for Astrophysics, 60 Garden St, Cambridge, MA 02138 USA

2 Australia Telescope National Facility, CSIRO, Epping, NSW 2121 Australia

3 Jet Propulsion Laboratory, 4800 Oak Grove Dr, Pasadena, CA 91109 USA

4 Raytheon, Canberra Deep Space Communications Complex, Tidbinbilla Rd, Paddy's Creek, ACT 2609, Australia

5 Instituto de Astrofísica de Andalucía, CSIC, Apartado 3004, E-18080 Granada, Spain

6 Madrid Deep Space Communication Complex, INTA-NASA, Paseo del Pintor Rosales 34-bajo, E-28008 Madrid, Spain

7 Laboratorio de Astrofísica Espacial y Física Fundamental, INTA, Apartado 50727, E-28080 Madrid, Spain

Electronic address: pkondrat@cfa.harvard.edu of the disk (high-velocity emission). Sources that display such spectra are referred to here as high-velocity systems and constitute $\sim 40 \%$ of the known nuclear water masers.

The study of high-velocity systems with VLBI has significantly contributed to our understanding of the immediate vicinity (i.e., $\lesssim 1 \mathrm{pc}$ ) of supermassive black holes. In three of these systems - NGC 4258 (Mivoshi et al. 1995), NGC 1068 (Greenhill \& Gwinn 1997), and the Circinus Galaxy (Greenhill et al. 2003) — resolved position and line-of-sight velocity data provided evidence for differential rotation and enabled accurate estimation of black hole mass and pc-scale molecular disk structure. Another system, NGC 3079, in which the rotation curve traced by the maser emission appears flat, was interpreted in the context of a pc-scale, thick, edgeon, self-gravitating, and possibly star forming molecular disk (Kondratko et al. 2005; Yamauchi et al. 2004). In addition to mapping pc-scale molecular disk structure and accurately weighing supermassive black holes, nuclear water maser emission has also been used as a distance indicator. Distance determination is possible for systems where a robust pc-scale disk model from VLBI maps is combined with a measurement of either maser proper motions or drifts in line-of-sight velocity of spectral features (i.e., centripetal acceleration). The distance to NGC 4258 obtained in this manner is the most accurate extragalactic distance thus far, is independent of standard candle calibrators such as Cepheids (Herrnstein et al. 1999), and it has contributed to analysis of the Cepheid period-luminosity relation (Freedman et al. 2001; Newman et al. 2001).

The $\sim 60$ known nuclear water masers are in the great majority of cases associated with Seyfert $1.8-2.0$ or LINER nuclei; only five systems are exceptions: NGC 5506 (NLSy1 from Nagar et al. 2002; Braatz et al. 1996), NGC 4051 (Sy1.5 from the NED; Hagiwara et al. 2003), NGC 2782 (Sy1, starburst from the NED; 
Braatz et al. 2004), NGC 4151 (Sy1.5 from the NED; Braatz et al. 2004), and 3C 403 (FRII; Tarchi et al. 2003). In the context of the unified AGN model, Seyfert $1.8-2.0$ systems contain an active nucleus and an obscuring structure along the line of sight to the central engine (Lawrence \& Elvis 1982; Antonucci 1993). Irradiation of molecular gas by X-rays from the central engine is a plausible means of exciting maser emission (e.g., Neufeld. Malonev. \& Conger 1994), which might explain the association of maser emission with nuclear activity in general. Maser emission might be associated with Seyfert $1.8-2.0$ systems in particular because, over a range of AGN luminosity, the shielding column density that provides the obscuring geometry maintains not only a reservoir of molecular gas but also physical conditions conducive to maser action, which are temperatures of $250-1000 \mathrm{~K}$ and $\mathrm{H}_{2}$ number densities of $10^{8-10} \mathrm{~cm}^{-3}$ (e.g., Desch, Wallin. \& Watson 1998). Since there is good evidence for a physical relationship between LINER systems and the AGN phenomenon (e.g., Ho et al. 1997b; $\mathrm{Ho}$ 1999; Ho et al. 2003), the distinction between these two types of activity in the context of maser emission might simply be one of luminosity, whereby a large fraction of LINERs correspond to low-luminosity analogues of Seyfert $1.8-2.0$ systems.

Since water maser emission is typically weak (« $0.1 \mathrm{Jy}$ ) and the velocity range of emission is determined by the orbital velocity of the molecular disk $\left(\gg 100 \mathrm{~km} \mathrm{~s}^{-1}\right)$, surveys designed to detect new water maser sources require both large, sensitive apertures and wide bandwidth spectrometers. The detection rate of water maser emission among Seyfert 2 and LINER galaxies with $v_{\text {sys }}<7000 \mathrm{~km} \mathrm{~s}^{-1}$ is $\sim 4 \%$ for surveys with a typical rms noise level on each source of $\sim 60 \mathrm{mJy}$ (thereafter, sensitivity; Braatz et al. 1996). This low detection rate, the weakness of the emission, and its potential wide velocity range make the discovery of new water maser sources challenging. Most past survey work has been characterized by limited sensitivity $(\sim 60 \mathrm{mJy})$ and narrow bandwidths $\left(\sim 700 \mathrm{~km} \mathrm{~s}^{-1}\right)$ and thus might have missed new maser sources because the emission was either too weak or outside the observing bands. These limitations of the previous surveys provide the major impetus for the present work. We note that a recent survey with $\sim 3$ mJy sensitivity (converted to $1.3 \mathrm{~km} \mathrm{~s}^{-1}$ spectral channels) using the Green Bank Telescope (GBT) has yielded a detection rate of $\sim 20 \%$ among Seyfert 2 and LINER systems with $v_{\text {sys }}<7500 \mathrm{kms}^{-1}$, though it was limited to just 145 sources (Braatz et al. 2004).

In order to discover more high-velocity systems, we procured a custom-built 4096-channel spectrometer with $5300 \mathrm{~km} \mathrm{~s}^{-1}$ bandwidth and are conducting a survey with the 70-m NASA Deep Space Network (DSN) antennas at Tidbinbilla, Australia and at Robledo, Spain. We selected our sample from among 1150 AGN with $v_{\text {sys }}<$ $14600 \mathrm{~km} \mathrm{~s}^{-1}$ listed in the NASA Extragalactic Database (NED), with preference for Seyfert 1.8 - 2.0 and LINER systems at lower recessional velocities. Thus far, we have discovered water maser emission in 15 AGN. The first seven discoveries were reported in Greenhill et al. (2003); here, we present spectra of eight most recent detections.

\section{OBSERVATIONS}

The discoveries reported here were obtained during the 2004-2005 northern winter with the Robledo 70-m antenna and during the 2003 and 2005 southern winters with the Tidbinbilla $70-\mathrm{m}$ antenna. The observing system at Tidbinbilla and its calibration was described in Greenhill et al. (2003). The observing system setup at Robledo was identical to that at Tidbinbilla. We estimated the gain curve and aperture efficiency of the Robledo antenna through measurement of opacity corrected antenna temperature of 3C 147, for which we adopted a flux density of $1.82 \mathrm{Jy}$ at $22.175 \mathrm{GHz}$ (Baars et al. 1977). The resulting peak efficiency was $0.43 \pm 0.09$ at $43^{\circ} \pm 5^{\circ}$ elevation, which yields a sensitivity of $1.7 \pm 0.3 \mathrm{Jy} \mathrm{K}^{-1}$.

To obtain single-polarization total-power spectra of each source, we moved the telescope every 30 or $45 \mathrm{~s}$ between the target source and a reference position on the sky $\sim 0.2^{\circ}$ away. Antenna rms pointing errors were typically $7^{\prime \prime}$ and system temperatures ranged from 40 to $75 \mathrm{~K}$ depending on elevation and weather. Typical $1 \sigma$ noise levels attained in an integration time (on+off source) of one hour were $\sim 8-17 \mathrm{mJy}$ in a $1.3 \mathrm{~km} \mathrm{~s}^{-1}$ channel. The spectra reported here have been Hanning smoothed to an effective resolution of $3.5 \mathrm{~km} \mathrm{~s}^{-1}$ and corrected for atmospheric opacity estimated from tipping scans.

\section{RESULTS}

During the 2003 and 2005 southern winters, we detected maser emission in six AGN with the Tidbinbilla antenna: AM 2158-380 NED02, IC 0184, NGC 0235A, NGC 0613, NGC 3393, and NGC 5495 (Fig. 11 and Table 11. Maser emission from NGC 4293 and VII ZW 073 was detected during the 2004-2005 northern winter with the Robledo antenna. Each discovery was confirmed by at least one observation with another instrument (Table 11). Positions of the maser emission measured with the Very Large Array (VLA) of the $\mathrm{NRAO}^{8}$ are consistent with optical positions of the AGN to within $1 \sigma(0 . \prime 3$ radio and 1.'3 optical; Table 1), which confirms the association of the discovered emission with nuclear activity.

The nuclei that are host to the detected maser emission are spectroscopically classified as Seyfert 2 or LINER in all but two cases (Table 11). Ambiguity remains in the case of NGC0613, which is listed as a possible Seyfert by Veron-Cetty \& Veron (1986), and in the case of NGC 0235A, which is classified as Seyfert 2 by Monk et al. (1986) but as Seyfert 1 by Maia et al. (1987). There is some indication of a broad component in $\mathrm{H} \alpha$ in the optical spectrum presented by Maia et al. (1987) (the Monk et al. (1986) spectrum does not cover the wavelength of $\mathrm{H} \alpha$ ). However, optical spectra presented by Monk et al. (1986) and Maia et al. (1987) exhibit a narrow $\mathrm{H} \beta$ line and a strong [O III] $\lambda 5007$ line relative to $\mathrm{H} \beta$. According to the quantitative classification scheme of Winkler (1992) (see also Osterbrock 1977, 1981), NGC 0235A thus harbors either a Seyfert 1.9 or a Seyfert 2 nucleus. A spectrum obtained independently by J. Huchra (2004, private communication) confirms this classification. It is thus likely that NGC 0235A was misclassified based on the hint of broad $\mathrm{H} \alpha$ reported by Maia et al. (1987).

8 The National Radio Astronomy Observatory is operated by Associated Universities, Inc., under cooperative agreement with the National Science Foundation 
The maser spectrum of NGC 3393 shows a characteristic spectral signature of emission from an edge-on disk: two high-velocity complexes $(\sim 70 \mathrm{mJy})$ symmetrically offset by $\sim 600 \mathrm{kms}^{-1}$ from the systemic velocity and a single spectral complex $(\sim 28 \mathrm{mJy})$ within $120 \mathrm{~km} \mathrm{~s}^{-1}$ of the systemic velocity. We have confirmed the weak systemic feature with the Tidbinbilla antenna $(1 \sigma=$ $7 \mathrm{mJy})$ and with the GBT $\left(1 \sigma=2 \mathrm{mJy}\right.$ in a $0.33 \mathrm{~km} \mathrm{~s}^{-1}$ spectral channel). If the high-velocity emission indeed originates from the midline of an edge-on disk, then the orbital velocity of the disk as traced by the maser emission is $\sim 600 \mathrm{~km} \mathrm{~s}^{-1}$, making NGC 3393 the third fastest known rotator after NGC $4258\left(\sim 1200 \mathrm{~km} \mathrm{~s}^{-1}\right.$; Mivoshi et al. 1995) and ESO269-G012 ( 650 $\mathrm{km} \mathrm{s}^{-1}$; Greenhill et al. 2003). Because the high-velocity emission extends over $\Delta v \sim 200 \mathrm{~km} \mathrm{~s}^{-1}$, it must occupy a fractional range of radii $\Delta r / R \approx 2 \Delta v / v=2 / 3$, which yields $\Delta r \sim 0.15-0.57 \mathrm{pc}$, assuming Keplerian rotation $\left(v \propto r^{-0.5}\right)$ and a range in disk sizes from that of NGC $4258(0.16-0.28 \mathrm{pc})$ to NGC $1068(0.6-1.1 \mathrm{pc})$. The corresponding central mass would be on the order of $5 \times 10^{7} M_{\odot}$ and the anticipated centripetal acceleration - that is the secular velocity drift of the systemic feature - would be on the order of $1 \mathrm{~km} \mathrm{~s}^{-1} \mathrm{yr}^{-1}$, which should be readily detectable within one year using single dish monitoring.

We confirmed the detection of high-velocity lines in NGC 5495 (refer to Fig. 1) with the GBT $(1 \sigma=5 \mathrm{mJy}$ in a $0.33 \mathrm{~km} \mathrm{~s}^{-1}$ spectral channel). If we again assume that the high-velocity emission originates from the midline of an edge-on disk, then the orbital velocity of the accretion disk is $\sim 400 \mathrm{~km} \mathrm{~s}^{-1}$ while the corresponding central mass and centripetal acceleration are on the order of $10^{7} M_{\odot}$ and $0.5 \mathrm{~km} \mathrm{~s}^{-1} \mathrm{yr}^{-1}$, respectively, assuming disk sizes as in NGC 4258 and NGC 1068.

Each of the remaining six detections - AM 2158380 NED02, IC 0184, NGC 0235A, NGC 0613, NGC 4293, and VII ZW 073 - displays only a single complex of spectral features. In particular, the spectrum of NGC 0613 reveals a very broad emission feature (full width at halfmaximum of $\sim 87 \mathrm{~km} \mathrm{~s}^{-1}$ ) and such broad lines have been typically associated with radio jets rather than molecular disks (e.g., Peck et al. 2003; Claussen et al. 1998). The spectral lines in NGC 0235A, VIIZW 073, and IC 0184 are significantly displaced from systemic velocities of the host galaxies (by $\sim 380, \sim 180$, and $\sim 220 \mathrm{kms}^{-1}$, respectively) and thus might be either emission features associated with radio jets (e.g., Peck et al. 2003; Claussen et al. 1998) or high-velocity emission lines from only a single side of an edge-on accretion disk. NGC 0613, NGC 3393, NGC 4293, and IC 0184 have been targeted but not detected in previous surveys for maser emission. For NGC 0613, Braatz et al. (1996) and Henkel et al. (1984) report $1 \sigma$ noise levels of $364 \mathrm{mJy}$ and $70 \mathrm{mJy}$ in 1.7 and $1.3 \mathrm{kms}^{-1}$ spectral channels, respectively, corresponding to signal-to-noise ratios of $\lesssim 1$ at the current line strength (peak flux of $\sim 70 \mathrm{mJy}$ in a $1.3 \mathrm{~km} \mathrm{~s}^{-1}$ spectral channel). Considering the large line-width, broader spectral averaging could have been applied to Henkel et al. (1984) data to achieve a marginal detection, and it is thus unclear whether this observation indicates line variability analogous to that observed in other masers associated with jet activity (e.g., Peck et al. 2003). For NGC 3393, Braatz et al.
(1996) report $1 \sigma$ noise level of $11 \mathrm{mJy}$ in $0.66 \mathrm{~km} \mathrm{~s}^{-1}$ channels, which should have been sufficient to detect the maser emission at its present strength of $\sim 80 \mathrm{mJy}$ (in a $1.3 \mathrm{~km} \mathrm{~s}^{-1}$ channel). In the case of NGC 4293, J.A. Braatz (private communication) obtained in 1998 a $1 \sigma$ noise level in a $1.3 \mathrm{~km} \mathrm{~s}^{-1}$ channel of $35 \mathrm{mJy}$, which would not have been sufficient to detect the weak emission (peak of $\sim 40 \mathrm{mJy}$ in a $1.3 \mathrm{~km} \mathrm{~s}^{-1}$ channel). For IC 0184, Braatz et al. (1996) report $1 \sigma$ noise level of $63 \mathrm{mJy}$ in $0.66 \mathrm{~km} \mathrm{~s}^{-1}$ channels, again not sufficient to detect the maser at present line strength (peak of $\sim 27 \mathrm{mJy}$ in a $1.3 \mathrm{~km} \mathrm{~s}^{-1}$ channel).

In a survey with the Tidbinbilla and Robledo antennas of $630 \mathrm{AGN}$ with $v_{\text {sys }}<14600 \mathrm{~km} \mathrm{~s}^{-1}$ selected from the NED (Table 2), we have detected to-date 15 new water maser sources (this paper and Greenhill et al. 2003). Since previous searches for water maser emission reported detections mostly in Seyfert $1.8-2.0$ or LINER systems (e.g., Braatz et al. 1996), we have focused our survey primarily on obscured nuclei (488 Seyfert 1.8 - 2.0 or LINER systems, among which there are the 15 new maser sources, and 143 Seyfert 1.0 - 1.5 systems, for which we report no new detections). Our survey includes $55 \%$ of known AGN with $v_{\text {sys }}<14600 \mathrm{kms}^{-1}$ (630 out of 1150 ) and is nearly complete to $7000 \mathrm{~km} \mathrm{~s}^{-1}$ with $82 \%$ of Seyfert $1.8-2.0$ and LINER systems $(325$ out of 398) already observed, although AGN catalogs consolidated by the NED may themselves be incomplete. The detection rate among nearby $\left(v_{\text {sys }}<7000 \mathrm{~km} \mathrm{~s}^{-1}\right)$ Seyfert $1.8-2.0$ and LINER galaxies is $4 \%$. The detection rate among nearby Seyfert $1.8-2.0$ and LINER galaxies that have been observed with absolute sensitivity (i.e., $1 \sigma$ noise level in flux density units multiplied by the spectral channel width and converted to luminosity using $H_{o}=75 \mathrm{~km} \mathrm{~s}^{-1} \mathrm{Mpc}$ ) of better than $2 L_{\odot}$ is $4 \%$, consistent with the analogous detection rate of $6 \%$ reported by Braatz et al. (1996). However, taking into account all known maser sources with peak flux densities above four times the typical rms achieved in this survey $\left(4 \sigma=56 \mathrm{mJy}\right.$ or $15 L_{\odot}$ in a $1.3 \mathrm{~km} \mathrm{~s}^{-1}$ spectral channel at $v_{\text {sys }}=7000 \mathrm{~km} \mathrm{~s}^{-1}$ ), the resulting incidence rate of maser emission among nearby Seyfert $1.8-2.0$ and LINER systems is $\sim 10 \%$, which should be compared to the analogous incidence rates of $\sim 7 \%$ and $\sim 20 \%$ for surveys with $\sim 60 \mathrm{mJy}\left(\sim 1 \mathrm{~km} \mathrm{~s}^{-1}\right.$ spectral channels; Braatz et al. 1996) and $\sim 3$ mJy (converted to $1.3 \mathrm{~km} \mathrm{~s}^{-1}$ spectral channels; Braatz et al. 2004) sensitivities, respectively. Six of the detections obtained with DSN (NGC 0613, NGC 3393, NGC 4293, NGC 5643, NGC 6300, and IC 0184) lie in nuclei that had been targeted in previous surveys, which demonstrates the importance of either survey sensitivity or source variability. As a result of this work, the number of nearby AGN $\left(v_{\text {sys }}<7000 \mathrm{~km} \mathrm{~s}^{-1}\right)$ observed with $<20$ mJy sensitivity has increased from 130 to 449.

\section{FUTURE PROSPECTS}

Four of the fifteen detections obtained using the 70-m antennas of the DSN - NGC 3393, NGC 5495 (this study), ESO 269-G012, and possibly NGC 6926 (Greenhill et al. 2003) - display the archetypal spectral signature of emission from an edge-on disk. The remaining 11 sources appear to be non-high-velocity systems, but monitoring and deep integrations (as with the GBT, 
which is roughly an order of magnitude more sensitive than the DSN antennas) may result in detection of highvelocity features. All high-velocity systems stronger than few mJy are good candidates for follow-up high angular resolution study with VLBI, where pc-scale molecular disk geometry and black hole mass may be inferred directly from resolved position and line-of-sight velocity data with high accuracy and relatively few sources of systematic uncertainty. Assuming disk sizes of 0.1 to 1 pc (i.e., comparable to NGC 4258 and NGC 1068, respectively) and a VLBI resolution element of $\sim 0.3$ mas, the source structure should be readily resolved because the AGN are relatively nearby $\left(3704 \mathrm{~km} \mathrm{~s}^{-1}<v_{\text {sys }}<\right.$ $6589 \mathrm{~km} \mathrm{~s}^{-1}$ ). The additional detection with single dish spectroscopic monitoring of secular drift in the velocities of low-velocity Doppler components (i.e., centripetal acceleration) may also enable estimation of geometric distances, as has been done for NGC 4258 (Herrnstein et al. 1999). A geometric distance to any of these systems would be of considerable value since it might help in calibration of the Hubble relation independent of standard candles such as Cepheids.
We are grateful for the invaluable support provided by the management, operations, and technical staff of the Canberra and Madrid Deep Space Communications Complexes. We thank M. Franco for Radio Frequency support and most especially P. Wolken and the staff charged with scheduling antenna time, without whose help and guidance this project would have been impossible. This research has made an extensive use of the NASA/IPAC Extragalactic Database (NED) which is operated by the Jet Propulsion Laboratory (JPL), California Institute of Technology, under contract with NASA. This work was supported in part by R\&D funds of the Smithsonian Astrophysical Observatory. IdG and JFG are partially supported by grant AYA2002-00376 (FEDER funds) of the Ministerio de Educación y Ciencia (Spain). Canberra Deep Space Communication Complex is managed by Commonwealth Scientific and Industrial Research Organisation (CSIRO) on behalf of JPL for NASA, with operations and maintenance undertaken by Raytheon, Australia. The Australia Telescope National Facility is funded by the Commonwealth Government for operation as a national facility by the CSIRO.

\section{REFERENCES}

Antonucci, R. 1993, ARA\&A, 31, 473

Baan, W. A., \& Haschick, A. 1996, ApJ, 473, 269

Baars, J. W. M., Genzel, R., Pauliny-Toth, I. I. K., \& Witzel, A. 1977, A\&A, 61, 99

Braatz, J., Greenhill, L., Moran, J., Wilson, A., \& Herrnstein, J. 1997, Bulletin of the American Astronomical Society, 29, 1374

Braatz, J. A., Henkel, C., Greenhill, L. J., Moran, J. M., \& Wilson, A. S. 2004, ApJ, 617, L29

Braatz, J. A., Wilson, A. S., \& Henkel, C. 1996, ApJS, 106, 51

Claussen, M. J., Diamond, P. J., Braatz, J. A., Wilson, A. S., \& Henkel, C. 1998, ApJ, 500, L129

Desch, S. J., Wallin, B. K., \& Watson, W. D. 1998, ApJ, 496, 775 Freedman, W. L., et al. 2001, ApJ, 553, 47

Greenhill, L. J., \& Gwinn, C. R. 1997, Ap\&SS, 248, 261

Greenhill, L. J., Kondratko, P. T., Lovell, J. E. J., Kuiper, T. B. H., Moran, J. M., Jauncey, D. L., \& Baines, G. P. 2003, ApJ, 582, L11

Greenhill, L. J., Moran, J. M., \& Herrnstein, J. R. 1997, ApJ, 481, L23

Greenhill, L. J., et al. 2003, ApJ, 590, 162

Hagiwara, Y., Diamond, P. J., Miyoshi, M., Rovilos, E., \& Baan, W. 2003, MNRAS, 344, L53

Hagiwara, Y., Diamond, P. J., Nakai, N., \& Kawabe, R. 2001, ApJ, 560,119

Henkel, C., Guesten, R., Downes, D., Thum, C., Wilson, T. L., \& Biermann, P. 1984, A\&A, 141, L1

Herrnstein, J. R., et al. 1999, Nature, 400, 539

Ho, L. C. 1999, Advances in Space Research, 23, 813

Ho, L. C., Filippenko, A. V., \& Sargent, W. L. W. 1997a, ApJS, 112,315

-. 1997b, ApJ, 487, 568

-. 2003, ApJ, 583, 159

Ishihara, Y., Nakai, N., Iyomoto, N., Makishima, K., Diamond, P., \& Hall, P. 2001, PASJ, 53, 215

Jungwiert, B., Combes, F., \& Axon, D. J. 1997, A\&AS, 125, 479
Kirhakos, S. D., \& Steiner, J. E. 1990, AJ, 99, 1722

Kondratko, P. T., Greenhill, L. J., \& Moran, J. M. 2005, ApJ, 618, 618

Lawrence, A., \& Elvis, M. 1982, ApJ, 256, 410

Maia, M. A. G., da Costa, L. N., Willmer, C., Pellegrini, P. S., \& Rite, C. 1987, AJ, 93, 546

Miyoshi, M., Moran, J., Herrnstein, J., Greenhill, L., Nakai, N., Diamond, P., \& Inoue, M. 1995, Nature, 373, 127

Monk, A. S., Penston, M. V., Pettini, M., \& Blades, J. C. 1986, MNRAS, 222, 787

Nagar, N. M., Oliva, E., Marconi, A., \& Maiolino, R. 2002, A\&A, 391, L21

Neufeld, D. A., Maloney, P. R., \& Conger, S. 1994, ApJ, 436, L127

Newman, J. A., Ferrarese, L., Stetson, P. B., Maoz, E., Zepf, S. E.,

Davis, M., Freedman, W. L., \& Madore, B. F. 2001, ApJ, 553, 562

Osterbrock, D. E. 1977, ApJ, 215, 733

一. 1981, ApJ, 249, 462

Peck, A. B., Henkel, C., Ulvestad, J. S., Brunthaler, A., Falcke, H., Elitzur, M., Menten, K. M., \& Gallimore, J. F. 2003, ApJ, 590, 149

Tarchi, A., Henkel, C., Chiaberge, M., \& Menten, K. M. 2003, A\&A, 407, L33

Trotter, A. S., Greenhill, L. J., Moran, J. M., Reid, M. J., Irwin, J. A., \& Lo, K. 1998, ApJ, 495, 740

Tully, R. B. 1988, Nearby galaxies catalog (Cambridge and New

York, Cambridge University Press, 1988, 221 p.)

Véron-Cetty, M.-P., \& Véron, P. 2003, A\&A, 412, 399

Veron-Cetty, M.-P., \& Veron, P. 1986, A\&AS, 66, 335

Winkler, H. 1992, MNRAS, 257, 677

Yamauchi, A., Nakai, N., Sato, N., \& Diamond, P. 2004, PASJ, 56, 605 

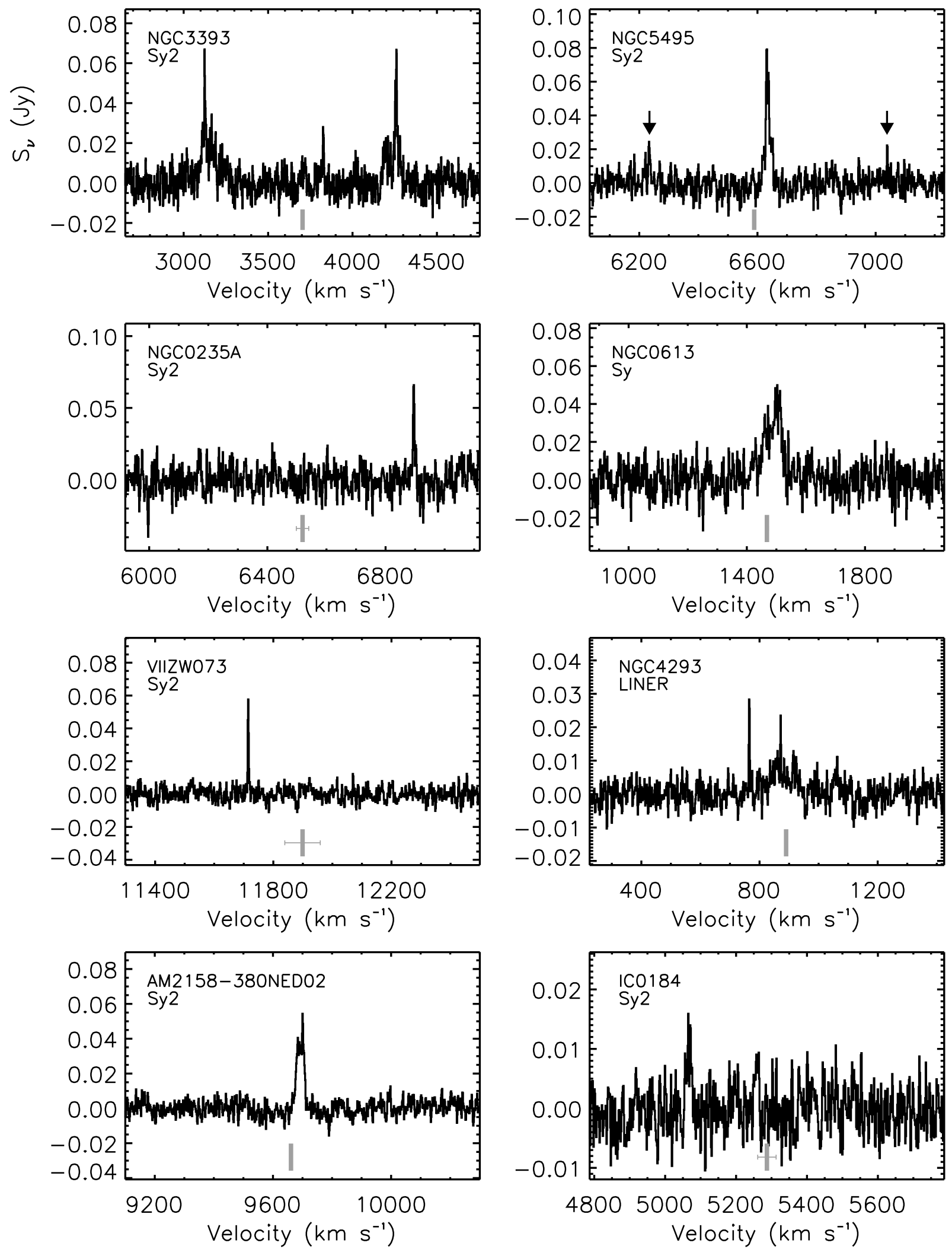

FIG. 1.- Spectra of NGC 3393, NGC 5495, NGC 0235A, NGC 0613, VII ZW 073, NGC 4293, AM 2158-380 NED02, and IC 0184 obtained with the 70-m Deep Space Network antennas at Tidbinbilla, Australia and Robledo, Spain. The arrows indicate NGC 5495 high-velocity features confirmed with the GBT. The velocity was computed in accordance with the radio definition of Doppler Shift and in the heliocentric reference frame. Vertical bars depict systemic velocities of the host galaxies and respective uncertainties. 
TABLE 1

Newly Discovered Nuclear Masers.

\begin{tabular}{|c|c|c|c|c|c|c|c|c|c|}
\hline Galaxy & Type $^{(a)}$ & $\begin{array}{l}\alpha_{2000}{ }^{(\mathrm{b})} \\
(\mathrm{hhmmss})\end{array}$ & $\begin{array}{c}\delta_{2000}{ }^{(\mathrm{b})} \\
(\mathrm{ddmmss})\end{array}$ & $\begin{array}{c}\mathrm{v}_{\text {sys }}(\mathrm{c}) \\
\left(\mathrm{km} \mathrm{s}^{-1}\right)\end{array}$ & $\begin{array}{c}\log _{10} L_{\mathrm{H}_{2} \mathrm{O}}(\mathrm{d}) \\
\left(\log _{10} L_{\odot}\right)\end{array}$ & $\begin{array}{l}\operatorname{Date}^{(e)} \\
(\text { ddd-yy) }\end{array}$ & $\begin{array}{c}T^{(\mathrm{f})} \\
(\mathrm{s})\end{array}$ & $\begin{array}{l}1 \sigma^{(\mathrm{g})} \\
(\mathrm{mJy})\end{array}$ & $\operatorname{Misc}^{(h)}$ \\
\hline NGC 0235A & Sy2 & $\begin{array}{lll}00 & 42 & 52.81 \\
00 & 42 & 52.81\end{array}$ & 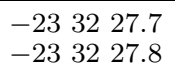 & 6519 & 2.0 & $214-03$ & 2280 & 15 & $\begin{array}{l}\text { TV } \\
\text { BnA }\end{array}$ \\
\hline NGC 0613 & Sy & $\begin{array}{llll}01 & 34 & 18.23 \\
01 & 34 & 18.18\end{array}$ & $\begin{array}{lll}-29 & 25 & 06.6 \\
-29 & 25 & 06.5\end{array}$ & 1468 & 1.2 & $174-03$ & 1680 & 16 & $\begin{array}{l}\text { TV } \\
\text { BnA }\end{array}$ \\
\hline IC 0184 & Sy2 & $\begin{array}{lll}01 & 59 & 51.23 \\
01 & 59 & 51.23\end{array}$ & $\begin{array}{lll}-06 & 50 & 25.4 \\
-06 & 50 & 25.4\end{array}$ & 5287 & 1.4 & $183-05$ & 8760 & 7 & $\begin{array}{l}\mathrm{GV} \\
\mathrm{CnB}\end{array}$ \\
\hline VII ZW 073 & Sy2 & $\begin{array}{llll}06 & 30 & 25.57 \\
06 & 30 & 25.54\end{array}$ & $\begin{array}{l}+634041.2 \\
+634041.3\end{array}$ & 11899 & 2.2 & $\begin{array}{l}337-04 \\
353-04\end{array}$ & 19560 & 6 & $\begin{array}{l}\text { GRV } \\
\text { B }\end{array}$ \\
\hline NGC 3393 & Sy2 & $\begin{array}{lll}10 & 48 & 23.46 \\
10 & 48 & 23.45\end{array}$ & $\begin{array}{lll}-25 & 09 & 43.4 \\
-25 & 09 & 43.6\end{array}$ & 3704 & 2.4 & $\begin{array}{l}167-03 \\
174-03\end{array}$ & 6120 & 8 & $\begin{array}{l}\text { GTV } \\
\text { BnA }\end{array}$ \\
\hline NGC 4293 & LINER & $\begin{array}{lll}12 & 21 & 12.89 \\
12 & 21 & 12.82\end{array}$ & $\begin{array}{l}+182256.6 \\
+182257.4\end{array}$ & 890 & $0.1-0.7$ & $\begin{array}{l}056-04 \\
063-04\end{array}$ & 15420 & 5 & $\begin{array}{l}\text { RV } \\
\text { B }\end{array}$ \\
\hline NGC 5495 & Sy2 & $\begin{array}{lll}14 & 12 & 23.35 \\
14 & 12 & 23.35\end{array}$ & $\begin{array}{l}-270628.9 \\
-2706 \\
29.2\end{array}$ & 6589 & 2.3 & $215-03$ & 2820 & 10 & $\begin{array}{l}\mathrm{GV} \\
\mathrm{BnA}\end{array}$ \\
\hline AM 2158-380 NED02 & Sy2 & $\begin{array}{llll}22 & 01 & 17.07 \\
22 & 01 & 17.10\end{array}$ & $\begin{array}{l}-374624.0 \\
-374623.0\end{array}$ & 9661 & 2.7 & $\begin{array}{l}175-05 \\
178-05\end{array}$ & 11460 & 7 & $\begin{array}{l}\text { GTV } \\
\text { CnB }\end{array}$ \\
\hline
\end{tabular}

(a) Activity type. NGC 5495 is classified as Seyfert 2 by Kirhakos \& Steiner (1990), NGC 3393, IC 0184, VII ZW 073, and AM 2158380 NED02 are listed as Seyfert 2 in the Veron-Cetty catalogue (Véron-Cettv \& Véron 2003), NGC 0613 is classified as a possible Seyfert by Veron-Cetty \& Veron (1986), NGC 4293 is listed as LINER by Ho et al. (1997a), while NGC 0235A is classified as Seyfert 1 by Maia et al. (1987) but as Seyfert 2 by Monk et al. (1986). Refer to the text for the discussion of the ambiguity in NGC 0235A spectral classification.

(b) First line: optical positions from the NED with uncertainties of $\pm 11^{\prime \prime} 3$. Second line: maser positions measured with a VLA snapshot with typical uncertainties of $\pm 00^{\prime \prime} 3$. In an effort to test for systematic errors in the derived maser positions, we imaged disjoint segments of the VLA data and confirmed that they yield consistent maser positions (that is, within $0{ }^{\prime \prime} 3$ ).

(c) Heliocentric systemic velocity computed assuming the radio definition of Doppler shift.

(d) Total water maser luminosity assuming isotropic emission of radiation and distances based on $H_{o}=75 \mathrm{~km} \mathrm{~s}^{-1} \mathrm{Mpc}^{-1}$, except for for NGC 0613 and NGC 4293, whose distances were adopted to be 17.9 Mpc (Jungwiert et al. 1997) and 17 Mpc (Tully 1988), respectively.

(e) Data obtained at listed epochs were combined to form a single spectrum.

(f) Total integration time on+off source.

(g) Rms noise in a $1.3 \mathrm{~km} \mathrm{~s}^{-1}$ spectral channel, corrected for atmospheric opacity (typically $\sim 0.07$ ) and for the dependence of antenna gain on elevation.

(h) First line: Instruments used to confirm the initial detections: $\mathrm{G}=\mathrm{GBT}, \mathrm{R}=$ Robledo, $\mathrm{T}=$ Tidbinbilla, V=VLA. The velocities and flux densities of the narrow spectral peaks in AM 2158-380 NED02, NGC 0235A, NGC 3393, NGC 5495, and NGC 4293 detected with the VLA agree with those measured using the Tidbinbilla antenna to within $1.3 \mathrm{~km} \mathrm{~s}^{-1}$ and $30 \%$, respectively. On the other hand, a $6 \mathrm{~km} \mathrm{~s}^{-1}$ discrepancy in the velocity centroid of the NGC 0613 spectral-line likely reflects uncertainty due to noise in the Tidbinbilla spectrum. Although the peak flux densities of the IC 0184 maser agree to within $20 \%$, the velocity centroids are in disagreement by $5 \mathrm{~km} \mathrm{~s}^{-1}$, which is again most likely due to low signal-to-noise ratio in the Tidbinbilla spectrum. The strengths of the VII ZW 073 maser measured with the VLA and the Robledo antenna differ by a factor of 2.5 (time baseline of 5 months), which could be due to either maser variability or low signal-to-noise ratio in both spectra. We note that such variability is not unusual (e.g., Baan \& Haschick 1996). The flux densities of AM 2158-380 NED02, IC 0184, NGC 3393, NGC 5495, and VII ZW 073 measured with the DSN and the GBT antennae agree to within 25\%. (The GBT spectra will be presented in a follow-up article.) Second line: Configuration of the VLA. The VLA bandwidth was $6.25 \mathrm{MHz}$ (channel spacing of $1.3 \mathrm{~km} \mathrm{~s}^{-1}$ ) for all imaged sources except NGC 0613, where a wider bandwidth of $25 \mathrm{MHz}$ (channel spacing of $11 \mathrm{~km} \mathrm{~s}^{-1}$ ) was necessary in order to include all the emission. 
TABLE 2

Sources Surveyed for Water Maser Emission with 70-m Deep Space Network Antennas at

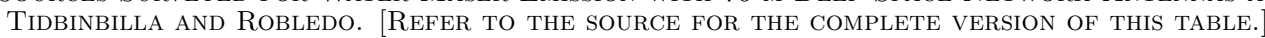

\begin{tabular}{lllcrcrrr}
\hline \hline Galaxy Name & Type $^{(\mathrm{a})}$ & $\begin{array}{c}\alpha_{2000}{ }^{(\mathrm{a})} \\
\text { (hhmmss) }\end{array}$ & $\begin{array}{c}\delta_{2000}{ }^{(\mathrm{a})} \\
(\mathrm{ddmmss})\end{array}$ & $\begin{array}{c}\mathrm{v}_{\text {sys }}{ }^{(\mathrm{a})} \\
\left(\mathrm{km} \mathrm{s}^{-1}\right)\end{array}$ & Date & $\begin{array}{c}T^{(\mathrm{b})} \\
(\mathrm{s})\end{array}$ & $\begin{array}{r}1 \sigma^{(\mathrm{c})} \\
(\mathrm{mJy})\end{array}$ & Site $^{(\mathrm{d})}$ \\
\hline KUG2358+330 & SY2 & 000058.14 & +332038.1 & 12387 & $2005-01-05$ & 6240 & 9 & $\mathrm{R}$ \\
UGC12914 & LINER & 000138.32 & +232901.5 & 4308 & $2003-02-03$ & 2910 & 18 & $\mathrm{R}$ \\
UGC12915 & LINER & 000141.94 & +232944.5 & 4274 & $2004-07-20$ & 2460 & 12 & $\mathrm{~T}$ \\
CGCG517-014 & SY2 & 000158.46 & +363856.6 & 9311 & $2004-12-26$ & 6060 & 9 & $\mathrm{R}$ \\
NGC7814 & LINER & 000314.89 & +160843.5 & 1046 & $2002-08-15$ & 1980 & 14 & $\mathrm{~T}$ \\
UGC00013 & LINER & 000329.23 & +272105.9 & 7498 & $2002-12-25$ & 2100 & 23 & $\mathrm{R}$ \\
\hline
\end{tabular}

(a) Type, position, and heliocentric systemic velocity obtained from the NED at the outset of the survey in 2002. Velocities are computed assuming the radio definition of Doppler shift.

(b) Total integration time on+off source.

(c) Rms noise in a $1.3 \mathrm{~km} \mathrm{~s}^{-1}$ spectral channel, corrected for atmospheric opacity (typically $\sim 0.07$ ) and for the dependence of antenna gain on elevation.

(d) $\mathrm{R}=$ Robledo, $\mathrm{T}=$ Tidbinbilla. 